\title{
PREDICTION OF DIAMETRAL CREEP FOR PRESSURE TUBES OF A PRESSURIZED HEAVY WATER REACTOR USING DATA BASED MODELING
}

\author{
JAE YONG LEE ${ }^{1}$ and MAN GYUN NA ${ }^{2 *}$ \\ ${ }^{1}$ Central Research Institute, Korea Hydro \& Nuclear Power Company \\ 70-1312-gil, Yuseong-daero, Yuseong-gu, Daejon 305-343, Republic of Korea \\ ${ }^{2}$ Department of Nuclear Engineering, Chosun University \\ 375 Seosuk-dong, Dong-gu, Gwangju 501-759, Republic of Korea \\ *Corresponding author. E-mail : magyna@chosun.ac.kr \\ Invited September14, 2011 \\ Received October 31, 2011 \\ Accepted for Publication February 27, 2012
}

The aim of this study was to develop a bundle position-wise linear model (BPLM) to predict Pressure Tube (PT) diametral creep employing the previously measured PT diameters and operating conditions. There are twelve bundles in a fuel channel, and for each bundle a linear model was developed by using the dependent variables, such as the fast neutron fluences and the bundle coolant temperatures. The training data set was selected using the subtractive clustering method. The data of 39 channels that consist of 80 percent of a total of 49 measured channels from Units 2, 3, and 4 of the Wolsung nuclear plant in Korea were used to develop the BPLM. The data from the remaining 10 channels were used to test the developed BPLM. The BPLM was optimized by the maximum likelihood estimation method. The developed BPLM to predict PT diametral creep was verified using the operating data gathered from Units 2, 3, and 4. Two error components for the BPLM, which are the epistemic error and the aleatory error, were generated. The diametral creep prediction and two error components will be used for the generation of the regional overpower trip setpoint at the corresponding effective full power days. The root mean square (RMS) errors were also generated and compared to those from the current prediction method. The RMS errors were found to be less than the previous errors.

KEYWORDS : CANDU Pressure Tube Creep, Linear Prediction Model, Maximum Likelihood Estimator, Uncertainty Analysis

\section{INTRODUCTION}

Diametral creep of the pressure tube (PT) is one of the principal aging mechanisms governing the heat transfer and hydraulic degradation of a heat transport system (HTS). The HTS of CANDU nuclear reactors experiences timedependent dimensional changes in the PTs through a creep mechanism. In particular, PT diametral creep leads to diametral expansion, which affects the thermal-hydraulic characteristics of the coolant channels and the critical heat flux (CHF). CHF is a key factor in the determination of the Critical Channel Power (CCP), which is in turn used in the calculation of the Regional Overpower Protection (ROP) system trip setpoints to ensure that the onset of intermittent fuel sheath dryout is prevented during slow Loss of Regulation (LOR) accidents. Therefore, it is imperative to predict the PT diametral creep in CANDU reactors, which is caused mainly by fast neutron irradiation, reactor coolant temperature, and so forth.

The currently used PT diametral creep prediction model considers the complex interactions between the effects of temperature and fast neutron flux on the deformation of PT zirconium alloys [1]. The model assumes long-term steady-state anisotropic deformation using the concepts of additive separable components of in-reactor thermal creep, irradiation-induced creep (shape change due to irradiation and applied stress at constant volume), and irradiation growth (shape change due to irradiation in the absence of applied stress at constant volume) [1]. This is a mechanistic model based on measured data. However, this model has high prediction uncertainty; in particular, when the variable Effective Full Power Days (EFPDs) is large, the root mean square (RMS) errors of the prediction of PT diameters rapidly increase.

Recently, a statistical error modeling method was developed using plant inspection data from the Bruce B CANDU reactor [2]. Owing to the significant effect of PT diametral creep on the safety margin in CANDU reactors, many studies have examined methods for its prediction. Notably, the Wolsung nuclear power plant has accumulated 
real plant inspection data including PT diameters. These data can be utilized to develop the PT diametral creep model. Also, Bruce B CANDU plants in Canada chose a data-based model instead of the conventional method for prediction of PT diameters.

There are two major operating parameters affecting PT diametral creep, the bundle average coolant temperature and the neutron fluences, especially fast neutron fluences having an energy level greater than $1 \mathrm{MeV}$ [3]. Fig.1 shows the effects of coolant temperatures and fast neutron

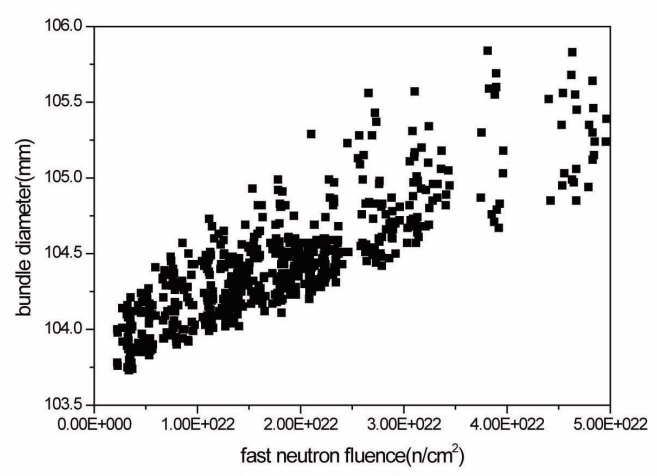

(a) Bundle diameter versus fast neutron fluence

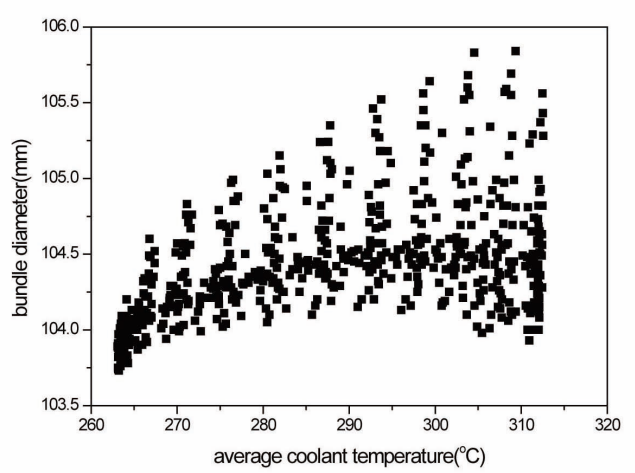

(b) Bundle diameter versus average coolant temperature

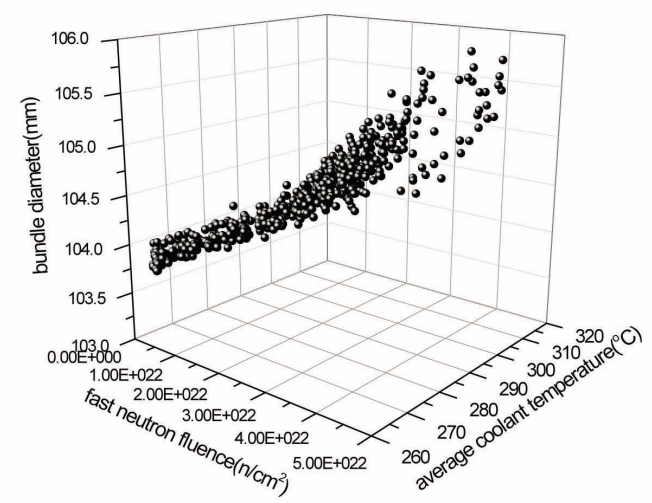

(c) $3 \mathrm{D}$ plot

Fig. 1. PT Diameter as a Function of Fast Neutron Fluence and Average Coolant Temperature fluencies on PT diametral creep [2]. Note that the PT diameter is observed to increase proportionally to increasing fluence and increasing temperature above the reference temperature.

The aim of this study was to develop a Bundle Positionwise Linear Model (BPLM) to predict PT diametral creep, employing previously measured PT diameters and HTS operating conditions. The BPLM was optimized by the Maximum Likelihood Estimation (MLE) method. The developed BPLM to predict PT diametral creep was verified using the operating data of nuclear power plants in Korea.

\section{BUNDLE POSITION-WISE LINEAR MODEL}

\subsection{Generation of a Linear Model}

A linear model for predicting the PT diametral creep can be generally described as follows:

$$
y_{i j}=a_{i j o}+a_{i 1} x_{i j 1}+a_{i 2} x_{i j 2}+\gamma_{i j}
$$

where $i$ indicates the bundle position in a PT channel and $j$ indicates the channel position. $x_{1}$ and $x_{2}$ are the normalized values of the input signals that represent the fast neutron fluence and average coolant temperature, respectively. $y$ is the normalized value of the differential diameter, $\omega_{i j}=\left(d_{i j}\right.$ $-d_{i j o}$ ) and $d_{i j}$ is the PT diameter at bundle position $i$ and channel $j$. $d_{i j o}$ is its initial diameter. Fig. 2 shows the fuel bundles in a CANDU reactor. The normalized values mean that they have a sample (measured channels) mean of zero and a sample standard deviation of one. For example,

$$
y_{i j}=\left(\omega_{i j}-m_{\omega}\right) / s_{\omega}
$$

where $m_{\omega}$ is the sample mean and $s_{\omega}$ is the sample standard deviation. The parameter $\gamma_{i j}$ indicates the measurement

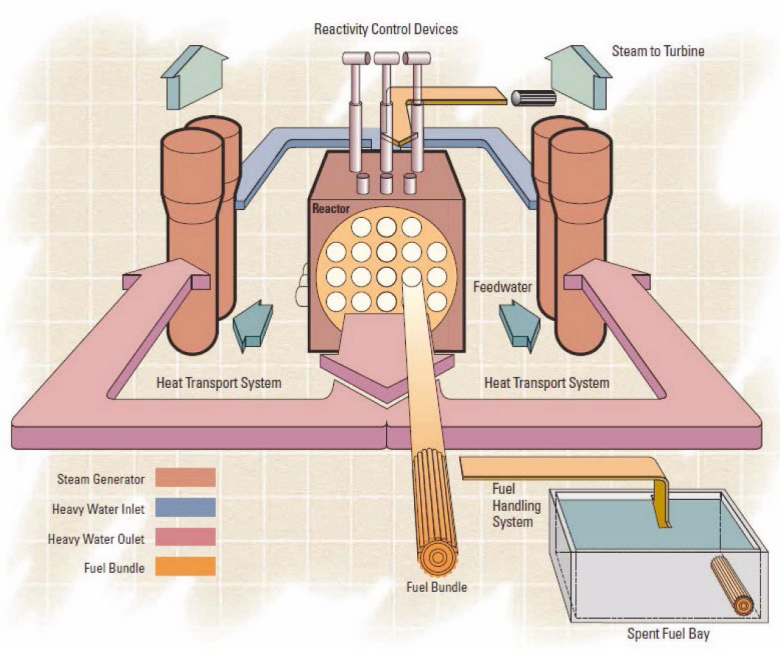

Fig. 2. Fuel Bundles in a CANDU Reactor System 
error, which is assumed to be independent and identically normally distributed with a mean zero and standard deviation $\sigma_{\gamma}$. Therefore, the true normalized differential diameter is as follows:

$$
y_{i j}^{t}=a_{i j o}+a_{i 1} x_{i j 1}+a_{i 2} x_{i j 2}
$$

Since the measured channels are assumed to be a random sample from the population of all 380 fuel channels in several EFPD conditions, the true normalized differential diameter $y_{i j}^{t}$ can be modeled as a random value as follows:

$$
y_{i j}^{t}=a_{i 0}+a_{i 1} x_{i j 1}+a_{i 2} x_{i j 2}+\delta_{j}
$$

where $a_{i 0}$ is a parameter that is common to the same bundle position of all channels and $\delta_{j}$ is independent and identically normally distributed with a mean zero and standard deviation $\sigma_{\delta} . \delta_{j}$ reflects the channel-to-channel variability and is called the aleatory error[4]. Thus, a BPLM was devised, because it is expected that the bundle position affects the diametral creep. The BPLM is described as follows:

$$
y_{i j}=a_{i 0}+a_{i 1} x_{i j 1}+a_{i 2} x_{i j 2}+\varepsilon_{i j}
$$

where $\varepsilon_{i j}=\delta_{j}+\gamma_{i j}$. A similar model was proposed previously [4]. However, the previous study [2] treated this $a_{i 0}$ as a single value regardless of the bundle position. The proposed BPLM uses a different $a_{i 0}$ according to the bundle position and employs the normalized input variables differently from the previous study. Also, the training data to be used to develop the BPLM are selected using the Subtractive Clustering (SC) scheme so that training data with much information will be used to develop a better model.

The estimated output (normalized differential diameter) from the BPLM at a specific EFPD condition is expressed as follows:

$$
\hat{y}_{i j}^{s}=\hat{a}_{i 0}+\hat{a}_{i j 1} x_{i j 1}^{s}+\hat{a}_{i j 2} x_{i j 2}^{s}
$$

where $\mathbf{x}_{i j}^{s}=\left[\begin{array}{lll}1 & x_{i j 1}^{s} & x_{i j 2}^{s}\end{array}\right]$

$x_{i j 1}^{s}=$ the normalized values of the fast neutron fluence

$x_{i j 2}^{s}=$ the normalized values of the average coolant temperature.

The best estimated PT diameter is

$$
\begin{aligned}
\hat{d}_{i j}^{s}=\left[s_{\omega} \hat{y}_{i j}^{s}+m_{\omega}\right]+d_{i j o} & \\
= & {\left[s_{\omega}\left(\hat{a}_{i 0}+\hat{a}_{i 1} x_{i j 1}^{s}+\hat{a}_{i 2} x_{i j 2}^{s}\right)+m_{\omega}\right]+d_{i j o} }
\end{aligned}
$$

The epistemic error in predicting the PT diameter at a given fixed condition $\mathbf{x}_{i j}^{s}$ can be written as[4]

$$
\begin{aligned}
\zeta_{i j}=d_{i j}^{s}-\hat{d}_{i j}^{s}=s_{\omega}\left(a_{i 0}+\right. & \left.a_{i 1} x_{i j 1}^{s}+a_{i 2} x_{i j 2}^{s}\right) \\
& -s_{\omega}\left(\hat{a}_{i 0}+\hat{a}_{i 1} x_{i j 1}^{s}+\hat{a}_{i 2} x_{i j 2}^{s}\right)=s_{\omega} \mathbf{x}_{i j}^{s}\left(\mathbf{a}_{i}-\hat{\mathbf{a}}_{i}\right)
\end{aligned}
$$

where $d_{i j}^{s}=\left[s_{\omega}\left(a_{i 0}+a_{i 1} x_{i j 1}^{s}+a_{i 2} x_{i j 2}^{s}\right)+m_{\omega}\right]+d_{i j o}$ is the true reference value, $\mathbf{x}_{i j}^{s}=\left[\begin{array}{lll}1 & x_{i j 1}^{s} x_{i j 2}^{s}\end{array}\right]$ and $\mathbf{a}_{i}^{T}=\left[\begin{array}{lll}a_{i 0} & a_{i 1} & a_{i 2}\end{array}\right]$.

The variance of the epistemic error is as follows [4]:

$$
V\left(\zeta_{i j}\right)=s_{\omega}{ }^{2} \mathbf{x}_{i j} \mathbf{\Omega} \mathbf{x}_{i j}^{T} \text { and } \boldsymbol{\Omega}=\operatorname{Var}\left(\mathbf{a}_{i}-\hat{\mathbf{a}}_{i}\right)=\left(\mathbf{X}^{T} \mathbf{V}^{-1} \mathbf{X}\right)^{-1}
$$

where $\mathbf{X}$ is the matrix of the measured diameter data and $\mathbf{V}$ is the variance matrix of $\varepsilon$ in Eq. (5).

Since the BPLM model is developed from a random sample from the population of all fuel channels, the true diameter at a random channel is written as

$$
d_{i j}=\left[s_{\omega}\left(y_{i j}^{s}+\delta\right)+m_{\omega}\right]+d_{i j o}=\left[s_{\omega} y_{i j}^{s}+m_{\omega}\right]+d_{i j o}+s_{\omega} \delta
$$

where $\delta$ is the aleatory error, which is independent and identically normally distributed with mean zero and standard deviation $\sigma_{\delta}$ and reflects the randomness in the true value of the PT diameters.

\subsection{Maximum Likelihood Estimation}

Since a CANDU PT channel has 12 bundles $(M=12)$, 12 BPLM are developed from $n(n=M \times J)$ training inputoutput data sets. $J$ indicates the total number of channels. The BPLM is described as follows, and is shown in Eq. (5)

$$
y_{i j}=a_{i 0}+a_{i 1} x_{i j 1}+a_{i 2} x_{i j 2}+\varepsilon_{i j}=\mathbf{x}_{i j} \mathbf{a}_{i}+\varepsilon_{i j} .
$$

Therefore, Eq. (11) can be rewritten as a matrix-vector form:

$$
\mathbf{y}=\mathbf{X} \boldsymbol{\alpha}+\varepsilon
$$

where

$$
\begin{aligned}
& \mathbf{y}^{T}=\left[\begin{array}{lllllll}
y_{11} & \cdots & y_{M 1} & \cdots & y_{1 J} & \cdots & y_{M J}
\end{array}\right]: n \times 1 \text { column vector } \\
& \boldsymbol{\alpha}^{T}=\left[\begin{array}{lllllllll}
a_{10} & \cdots & a_{M 0} & a_{11} & \cdots & a_{M 1} & a_{12} & \cdots & a_{M 2}
\end{array}\right]: 3 M \times 1 \text { colum } \\
& \mathbf{X}=\left[\begin{array}{lll}
\mathbf{x}_{o} & \mathbf{x}_{1} & \mathbf{x}_{2}
\end{array}\right]: n \times 3 M \text { matrix } \\
& \mathbf{x}_{o}^{T}=\left[\begin{array}{llll}
\operatorname{diag}\left(\mathbf{1}_{M \times 1}\right) & \operatorname{diag}\left(\mathbf{1}_{M \times 1}\right) & \cdots & \operatorname{diag}\left(\mathbf{1}_{M \times 1}\right)
\end{array}\right]: n \times M \text { matrix } \\
& \mathbf{x}_{1}^{T}=\left[\begin{array}{llll}
\operatorname{diag}\left(x_{\cdot 11}\right) & \operatorname{diag}\left(x_{\cdot 21}\right) & \cdots & \operatorname{diag}\left(x_{\cdot / 1}\right)
\end{array}\right]: n \times M \text { matrix } \\
& \mathbf{x}_{2}^{T}=\left[\begin{array}{llll}
\operatorname{diag}\left(x_{\bullet 12}\right) & \operatorname{diag}\left(x_{\cdot 22}\right) & \cdots & \operatorname{diag}\left(x_{\cdot J 2}\right)
\end{array}\right]: n \times M \text { matrix } \\
& \operatorname{diag}\left(x_{\cdot j k}\right)=\left[\begin{array}{llll}
x_{1 j k} & x_{2 j k} & \cdots & x_{M j k}
\end{array}\right] .
\end{aligned}
$$

Here, $\mathbf{1}_{M \times 1}$ denotes an $M \times 1$ matrix with all elements of ones.

The following likelihood function is used to solve the model coefficient $\boldsymbol{\alpha}$ with the covariance $\mathbf{V}$ [4]:

$$
L\left(y ; \boldsymbol{\alpha}, \sigma_{\delta}^{2}, \sigma_{\gamma}^{2}\right)=(2 \pi)^{-n / 2}|\mathbf{V}|^{-1 / 2} e^{\left(-\frac{1}{2}(\mathbf{y}-\mathbf{X} \boldsymbol{\alpha})^{T} \mathbf{V}^{-1}(\mathbf{y}-\mathbf{X} \boldsymbol{\alpha})\right)}
$$

where

$$
\begin{gathered}
\mathbf{V}=\mathbf{I}_{J} \otimes\left(\sigma_{\delta}^{2} \mathbf{1}_{M \times M}+\sigma_{\gamma}^{2} \mathbf{I}_{M}\right) \\
\mathbf{V}^{-1}=\frac{1}{\sigma_{\gamma}^{2}} \mathbf{I}_{J} \otimes\left(\mathbf{I}_{M}-\frac{\mathbf{1}_{M \times M}}{M+\kappa}\right) \\
\kappa=\sigma_{\gamma}^{2} / \sigma_{\delta}^{2} .
\end{gathered}
$$

The notation $\otimes$ denotes the tensor product. $\mathbf{1}_{M \times M}$ denotes an $M \times M$ matrix with all elements of ones. 
The log-likelihood function is defined as

$$
\begin{aligned}
l=\ln L\left(y ; \boldsymbol{\alpha}, \sigma_{\delta}^{2}, \sigma_{\gamma}^{2}\right)=-\frac{n}{2} & \ln (2 \pi) \\
& \quad-\frac{1}{2} \ln |\mathbf{V}|-\frac{1}{2}(\mathbf{y}-\mathbf{X} \boldsymbol{\alpha})^{T} \mathbf{V}^{-1}(\mathbf{y}-\mathbf{X} \boldsymbol{\alpha})
\end{aligned}
$$

From $\frac{\partial l}{\partial \boldsymbol{\alpha}}=\frac{\partial l}{\partial \sigma_{\delta}^{2}}=\frac{\partial l}{\partial \sigma_{\gamma}^{2}}=\mathbf{0}$, the maximum likelihood estimates of $\boldsymbol{\alpha}, \sigma_{\delta}^{2}$, and $\sigma_{\gamma}^{2}$ are as follows:

$$
\begin{gathered}
\hat{\boldsymbol{\alpha}}=\left(\mathbf{X}^{T} \mathbf{V}^{-1} \mathbf{X}\right)^{-1} \mathbf{X}^{T} \mathbf{V}^{-1} \mathbf{y} \\
\hat{\sigma}_{\delta}^{2}=\frac{(\mathbf{y}-\mathbf{X} \hat{\boldsymbol{\alpha}})^{T}\left(\mathbf{E}-\mathbf{I}_{n}\right)(\mathbf{y}-\mathbf{X} \hat{\boldsymbol{\alpha}})}{n(M-1)} \\
\hat{\sigma}_{\gamma}^{2}=\frac{(\mathbf{y}-\mathbf{X} \hat{\boldsymbol{\alpha}})^{T}\left(M \mathbf{I}_{n}-\mathbf{E}\right)(\mathbf{y}-\mathbf{X} \hat{\boldsymbol{\alpha}})}{n(M-1)}
\end{gathered}
$$

where $\mathbf{E}=\mathbf{I}_{J} \otimes \mathbf{1}_{M \times M}$. To express the variances of the measurement error and the aleatory error in PT diameters rather than the normalized value, a scale factor $s_{\omega}$ should be multiplied in Eqs. (19) and (20). That is, (y - Xô) should be modified into $s_{\omega}(\mathbf{y}-\mathbf{X} \hat{\alpha})$ in Eqs. (19) and (20).

The variances estimated by the maximum likelihood method are necessarily biased. The unbiased estimates of the variances can be solved by taking the expectation of the biased variances, Eqs. (19) and (20), as follows:

$$
\begin{gathered}
\hat{\sigma}_{\delta}^{2}=\frac{n(M-1) \hat{\sigma}_{\delta}^{2}}{n(M-1)-\left(\hat{\sigma}_{\gamma}^{2} / \hat{\sigma}_{\delta}^{2}\right)\left\{\operatorname{tr}\left[\left(\mathbf{E}-\mathbf{I}_{n}\right) \mathbf{X}\left(\mathbf{X}^{T}\left(\mathbf{I}_{n}-\frac{\mathbf{E}}{\left(\hat{\sigma}_{\gamma}^{2} / \hat{\sigma}_{\delta}^{2}\right)+M}\right) \mathbf{X}\right)^{-1} \mathbf{X}^{T}\right]\right\}} \\
\hat{\hat{\sigma}}_{\gamma}^{2}=\frac{n(M-1) \hat{\sigma}_{\gamma}^{2}}{n(M-1)-\operatorname{tr}\left[\left(M \mathbf{I}_{n}-\mathbf{E}\right) \mathbf{X}\left(\mathbf{X}^{T}\left(\mathbf{I}_{n}-\frac{\mathbf{E}}{\left(\hat{\sigma}_{\gamma}^{2} / \hat{\sigma}_{\delta}^{2}\right)+M}\right) \mathbf{X}\right)^{-1} \mathbf{X}^{T}\right]}
\end{gathered}
$$

The prediction interval (PI) with $95 \%$ confidence is defined as

$$
\begin{gathered}
P I_{i j}=\hat{y}_{i j} \pm t_{0.05 / 2}^{n-3 M}\left(\sqrt{\operatorname{Var}\left(\lambda_{i j}\right)}\right) \\
\operatorname{Var}\left(\lambda_{i j}\right)=\operatorname{diag}\left(\mathbf{x}_{\cdot j} \mathbf{\Omega} \mathbf{x}_{\cdot j}^{T}-\mathbf{x} \cdot \mathbf{x}_{j} \mathbf{\Omega}\left(\mathbf{X}^{T} \mathbf{V}^{-1}\right)\left(\sigma_{\delta}^{2} \mathbf{W} \mathbf{w}_{j}^{T}\right)\right. \\
\left.-\left(\sigma_{\delta}^{2} \mathbf{w}_{j} \mathbf{W}^{T}\right)\left(\mathbf{V}^{-1} \mathbf{X}\right) \mathbf{\Omega} \mathbf{x}_{\cdot j}^{T}+\sigma_{\delta}^{2} \mathbf{1}_{M \times M}+\sigma_{\gamma}^{2} \mathbf{I}_{M}\right)
\end{gathered}
$$

where $\boldsymbol{\Omega}=\left(\mathbf{X}^{T} \mathbf{V}^{-1} \mathbf{X}\right)^{-1}, \mathbf{W}=\mathbf{I}_{J} \otimes \mathbf{1}_{M \times 1}$ and $\mathbf{w}_{j}^{T}=\mathbf{I}_{J}(:, j) \times \mathbf{1}_{1 \times M}$.

The confidence interval (CI) is calculated using the variance of the epistemic errors of Eq. (9) as follows:

$$
C I_{i j}=\hat{y}_{i j} \pm t_{0.05 / 2}^{n-3 M}\left(\sqrt{V\left(\zeta_{i j}\right)}\right)
$$

From Eq. (12), the PT diameters are estimated by the following equation:

$$
\hat{\mathbf{y}}=\mathbf{X} \hat{\boldsymbol{\alpha}}
$$

The coefficient $\hat{\alpha}$ is calculated by Eq. (18) and Eq. (26) and is used to predict or estimate the PT diameters at a given fluence and coolant temperature.

\section{DATA PREPARATION}

The input-output data were normalized to be mean of zero and standard deviation of one before they were applied, which helps to accomplish the generalization of the input variables. Also, informative data should be used to develop the BPLM. In the following, the SC method to select the training data is introduced.

The appropriate selection of training data is very important, because the data can affect the optimization of the BPLM. The input and output training data are expected to be in the form of clusters and the data in these cluster centers are more informative than the neighboring data. A BPLM can be well trained using informative data.

It is assumed that $n$ input/output data $\left(\mathbf{z}_{k}=\left(\mathbf{x}_{k}, y_{k}\right), k=\right.$ $1,2, \ldots, n)$ are available and the data points are normalized in each dimension. The SC scheme begins by generating a number of clusters in $m \times n$ dimensional input space, where $m$ is the number of input variables. The SC scheme uses a measure of the potential of each data point, which is a function of the Euclidean distances to all other input data points [5]:

$$
P_{1}(k)=\sum_{j=1}^{n} e^{-4\left\|\mathbf{x}_{k}-\mathbf{x}_{j}\right\|^{2} / r_{\alpha}^{2}}, k=1,2, \ldots, n
$$

where $r_{\alpha}$ is the radius defining a neighborhood, which has considerable influence on the potential. Obviously, its potential is high when it is surrounded by a considerable amount of neighboring data. After the potential of each data point is calculated, the data point with the highest potential is selected as the first cluster center.

In general, after determining the $i^{\text {th }}$ cluster center $\mathbf{x}^{c}{ }_{i}$ and its potential value $P_{i}^{c}$, the potential of each data point is revised using the following equation:

$$
P_{i+1}(k)=P_{i}(k)-P_{i}^{c} e^{-4\left\|\mathbf{x}_{k}-\mathbf{x}_{i}^{\mathrm{c}}\right\|^{2} / r_{\beta}^{2}}, k=1,2, \ldots, n
$$

where $r_{\beta}$ is also the radius, which is usually greater than $r_{\alpha}$ so as to limit the number of generated clusters. When the potentials of all data points have been revised according to Eq. (28), the data point with the highest potential is selected as the next cluster center. These calculations stop if the inequality $P_{i}^{c}<\varepsilon P_{1}^{c}$ is true. Otherwise, they are repeated.

The data positioned at the cluster centers are used as selected training data to develop the BPLM model.

\section{VALIDATION OF THE DIAMETRAL CREEP PREDICTION MODEL}

The data used consisted of a total of 588 input-output data pairs taken from Units 2, 3, and 4 (U2, U3, and U4) of the Wolsung nuclear plant. These data were acquired at 1501, 1944, and 3256 effective full power days (EFPDs) from Unit 2, and 1324 and 2183 EFPDs from Unit 3, and 937 and 2154 EFPDs from Unit 4. In this study, all Units 
Table 1. Parameter Estimations of 12 BPLM Models

\begin{tabular}{c|c|c|c}
\hline Bundle position & $a_{i 0}$ & $a_{i 1}$ & $a_{i 2}$ \\
\hline 1 & 2.2172 & 0.1078 & 2.3428 \\
\hline 2 & 1.6243 & 0.4505 & 1.6468 \\
\hline 3 & 0.6178 & 0.5187 & 0.9638 \\
\hline 4 & 0.0620 & 0.5726 & 0.5416 \\
\hline 6 & -0.1707 & 0.6400 & 0.2226 \\
\hline 7 & -0.1264 & 0.7201 & 0.1653 \\
\hline 8 & -0.0223 & 0.8183 & 0.1478 \\
\hline 9 & 0.0126 & 0.9141 & 0.2613 \\
\hline 10 & 0.0354 & 1.0410 & 0.4150 \\
\hline 11 & 0.1006 & 1.1967 & 0.5332 \\
\hline 12 & 0.3816 & 1.3927 & 0.4824 \\
\hline
\end{tabular}

Table 2. Errors According to the Bundle Position

\begin{tabular}{c|c|c|c|c}
\hline \multirow{2}{*}{$\begin{array}{c}\text { Bundle } \\
\text { position }\end{array}$} & \multicolumn{2}{|c|}{ Training data } & \multicolumn{2}{c}{ Test data } \\
\cline { 2 - 5 }$(\mathrm{mm})$ & $\begin{array}{c}\text { Max. error } \\
(\mathrm{mm})\end{array}$ & $\begin{array}{c}\text { RMS error } \\
(\mathrm{mm})\end{array}$ & $\begin{array}{c}\text { Max. error } \\
(\mathrm{mm})\end{array}$ \\
\hline 1 & 0.0719 & 0.1827 & 0.0654 & 0.1316 \\
\hline 2 & 0.0840 & 0.2630 & 0.0716 & 0.1511 \\
\hline 3 & 0.0950 & 0.2988 & 0.0791 & 0.1460 \\
\hline 4 & 0.0975 & 0.2908 & 0.0799 & 0.1396 \\
\hline 5 & 0.0984 & 0.2732 & 0.0801 & 0.1510 \\
\hline 6 & 0.1039 & 0.2756 & 0.0821 & 0.1627 \\
\hline 7 & 0.1046 & 0.2793 & 0.0837 & 0.1666 \\
\hline 8 & 0.1085 & 0.2588 & 0.0933 & 0.1826 \\
\hline 9 & 0.1098 & 0.2426 & 0.0987 & 0.1926 \\
\hline 10 & 0.1155 & 0.2867 & 0.1009 & 0.2002 \\
\hline 11 & 0.1058 & 0.2822 & 0.0877 & 0.1915 \\
\hline 12 & 0.0867 & 0.1925 & 0.0680 & 0.1455 \\
\hline
\end{tabular}

Table 3. Uncertainties of the BPLM Model

\begin{tabular}{c|c|c|c|c|c}
\hline Data type & $\sigma_{\varepsilon}(\mathrm{mm})$ & $\sigma_{\delta}(\mathrm{mm})$ & $\sigma_{\gamma}(\mathrm{mm})$ & Epistemic error $(\mathrm{mm})$ & $\mathrm{RMS}$ error $(\mathrm{mm})$ \\
\hline Train data & 0.1032 & 0.0936 & 0.0394 & 0.0211 & 0.0992 \\
\hline Test data & 0.0995 & 0.0936 & 0.0394 & 0.0210 & 0.0833 \\
\hline
\end{tabular}

are considered to have the same type (material and composition) of pressure tubes. The data of 39 channels, which account for 80 percent of the total of 49 measured channels from Units 2, 3, and 4, were used to develop the BPLM. The channels of the training data were selected by the SC scheme. The data from the remaining 10 channels were used to test the developed BPLM models. The model parameter estimations of 12 BPLM models for each bundle position are given in Table 1 .

Table 2 provides the root mean square (RMS) errors and maximum errors according to the bundle positions. The error is the largest in bundle position 10. Table 3 summarizes the uncertainties of the BPLM. A testing dataset usually gives relatively larger errors than the training data set. Since the input/output data positioned in the cluster centers have more information and were used to develop the BPLM (training data), the data points that are slightly distant from the cluster centers are more likely to become the test data, which means that the data points are difficult to model. Therefore, the errors of the test data can be larger than those of the training data.
Table 4. Prediction Errors from BPLM and Current Design Method at 1501 EFPD

\begin{tabular}{c|c|c}
\hline odel & RMS error $(\mathrm{mm})$ & Max error $(\mathrm{mm})$ \\
\hline BPLM & 0.0681 & 0.1587 \\
\hline Current & 0.1503 & 0.2899 \\
\hline
\end{tabular}

The PT diameters estimated by the current design model were provided at 1500, 3000, 4500, 6000, and 7500 EFPDs, and the measured PT diameters were provided at some channels at 1501 EFPD (approximately 1500 EFPD), 1944, 3256, and so forth. Therefore, both the measured and estimated diameters (by the conventional method and the BPLM) were given at 1500 EFPD alone so that the prediction errors could be calculated. Table 4 provides the prediction errors from the BPLM and the current design method which is based on mechanistic phenomena of the diametral creep. According to the results provided in Table 4, the prediction errors are small compared to those 
from the current design method. Table 5 compares the uncertainties by the uncertainty analysis scheme that the current design method utilizes. The developed BPLM is superior to the current design model.
Fig. 3 shows the estimated diameters according to EFPD and 12 bundle positions at 4 different channels. This figure shows reasonable trends wherein the estimated diameter increases accordingly as the EFPD increases.

Table 5. Uncertainty Comparison by the Conventional Uncertainty Method for Wolsung Unit 2.

\begin{tabular}{c|c|c|c|c|c|c}
\hline \multirow{2}{*}{ EFPD } & \multicolumn{3}{|c|}{ BPLM } & \multicolumn{3}{c}{ Current Design Method } \\
\cline { 2 - 7 } & Sample Std. & $\begin{array}{c}\text { Estimated } \\
\text { population Std. }\end{array}$ & $\begin{array}{c}\text { Std. of sample } \\
\text { mean }\end{array}$ & Sample Std. & $\begin{array}{c}\text { Estimated } \\
\text { population Std. }\end{array}$ & $\begin{array}{c}\text { Std. of sample } \\
\text { mean }\end{array}$ \\
\hline 3000 & 7.67 & 11.26 & 3.56 & 11.16 & 17.53 & 6.20 \\
\hline 4350 & 8.69 & 12.77 & 4.04 & 11.38 & 17.89 & 6.32 \\
\hline 4500 & 8.85 & 13.00 & 4.11 & 11.38 & 17.89 & 6.32 \\
\hline 5500 & 9.83 & 14.44 & 4.57 & 11.46 & 18.01 & 6.37 \\
\hline 6500 & 10.63 & 15.62 & 4.94 & 11.46 & 18.01 & 6.37 \\
\hline 7500 & 11.27 & 16.56 & 5.24 & 11.45 & 17.99 & 6.36 \\
\hline
\end{tabular}

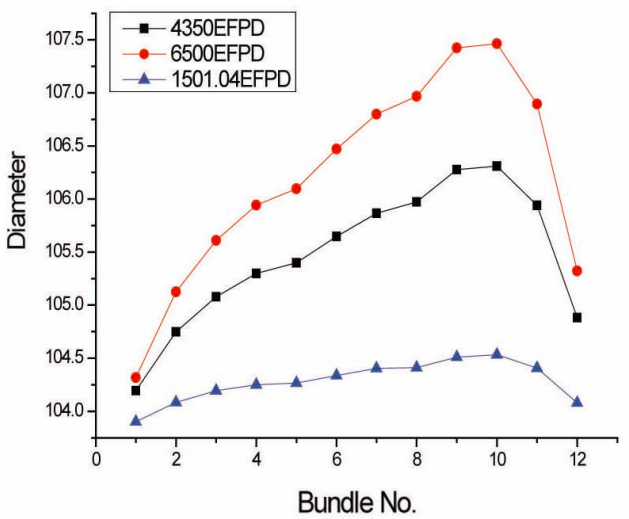

(a) Channel L09

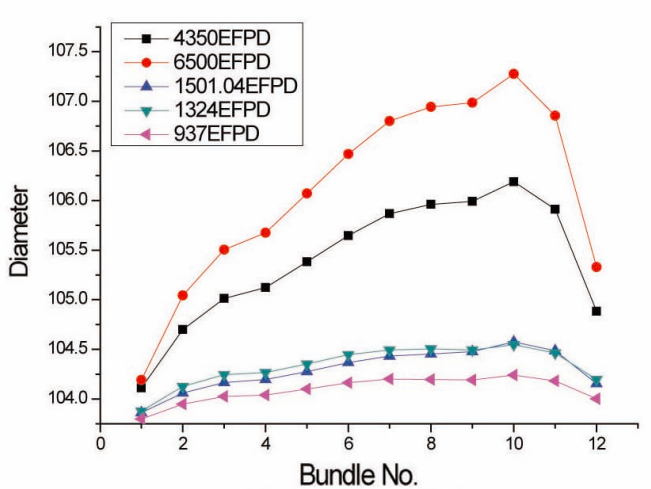

(c) Channel M11

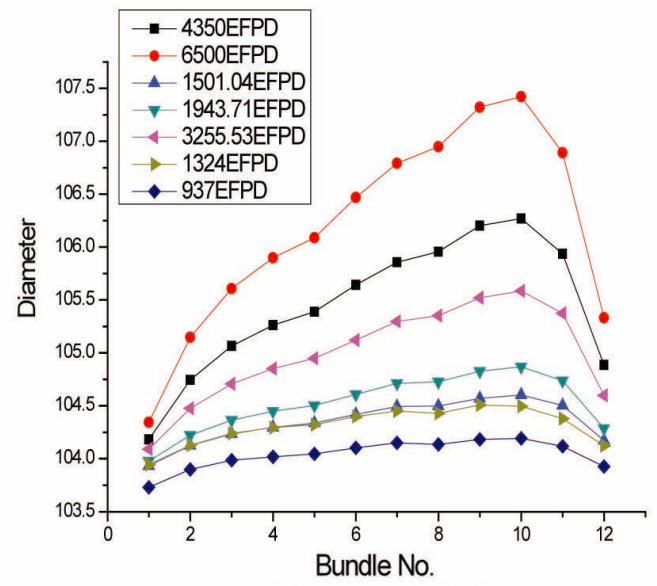

(b) Channel L13

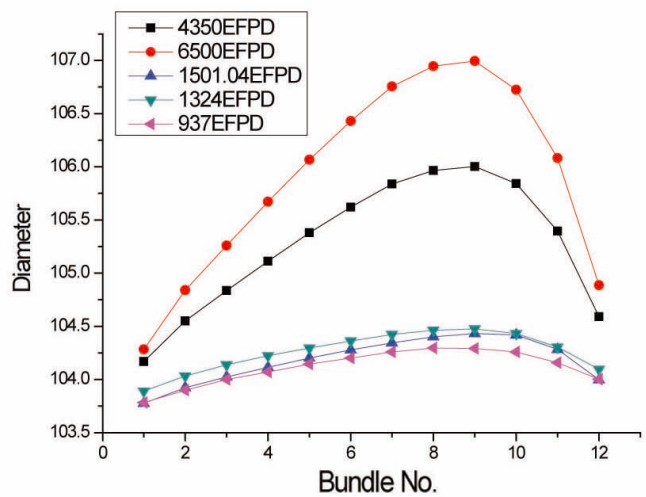

(d) Channel N03

Fig. 3. Estimated Diameters According to EFPD 
Fig. 4 shows the estimated channel-maximum PT diameters versus EFPD at channels A11 and L09. The proposed BPLM provides detailed estimations at an operating time

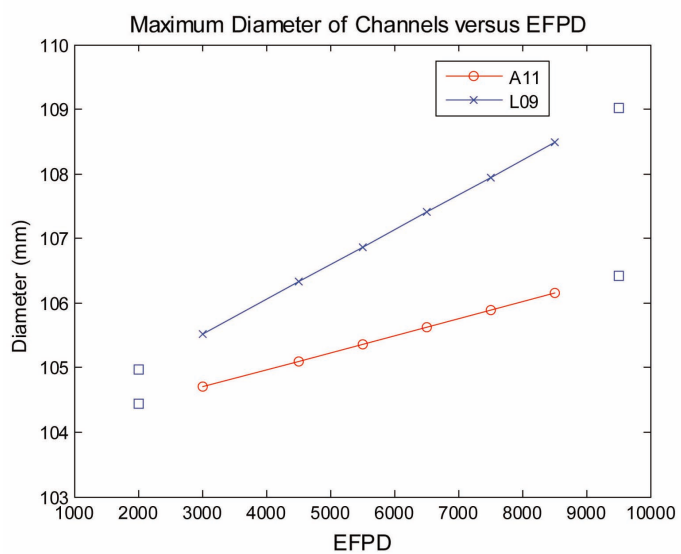

Fig. 4. Estimated Channel-Maximum PT Diameters versus EFPD at Channels A11 and L09

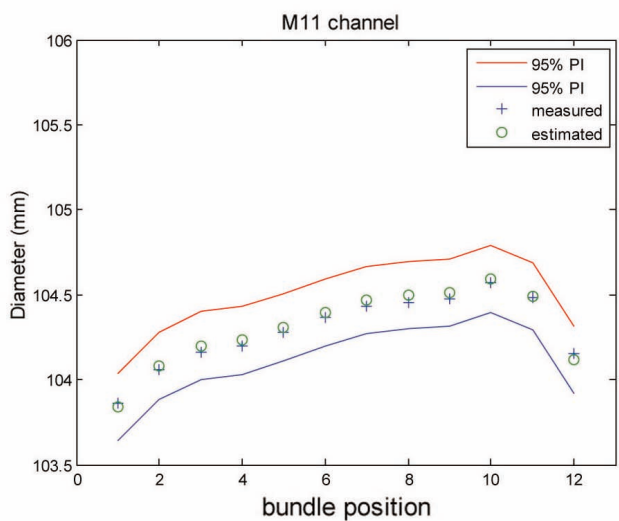

(a) M11 Channel (U2, 1501 EFPD)

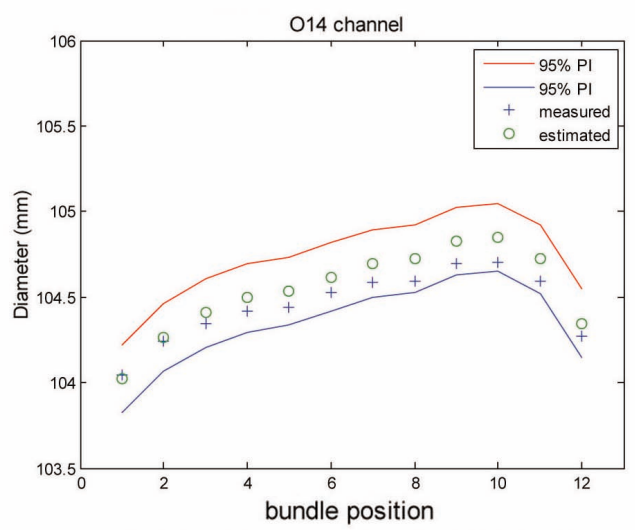

(c) O14 Channel, (U2, 1501 EFPD) point for specific channels. Fig. 5 shows the prediction intervals for test data, calculated by Eq. (23). Only one of 120 measured data points exceeds the prediction interval, meaning that $99.2 \%$ of the test data is covered by the prediction interval. The prediction interval is determined at a $95 \%$ confidence interval. It is known that the prediction interval has a sufficient margin. Fig. 6 shows the uncertainties as a function of the fast neutron fluence and the average coolant temperature. The epistemic errors are much higher at some fluences and coolant temperatures. These appear to be caused by measurement errors.

In this study, 49 channels of data were used for model training and testing. The BPLM, which is based on these data sets, can produce accurate estimated PT diameters, as shown in Tables 4 and 5. In the future, if more measurement data are taken from the plants, the model can be improved with these additional data. On the other hand, the conventional method cannot guarantee the current prediction accuracy in the future. For this reason, Bruce B plants chose a data-based model instead of the conventional method for prediction of PT diameters.

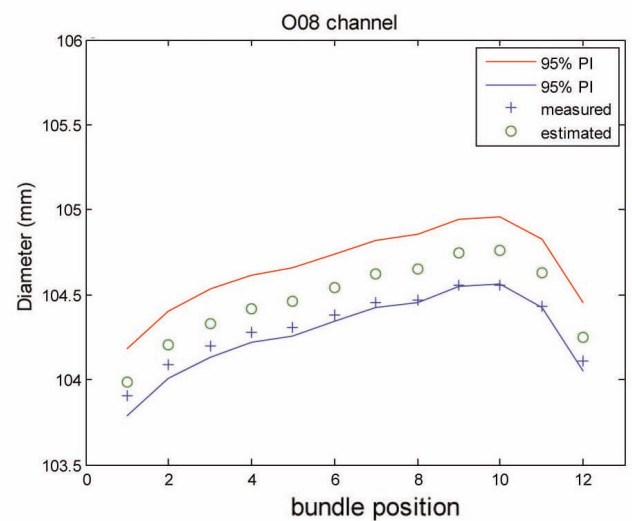

(b) O08 Channel (U2, 1501 EFPD)

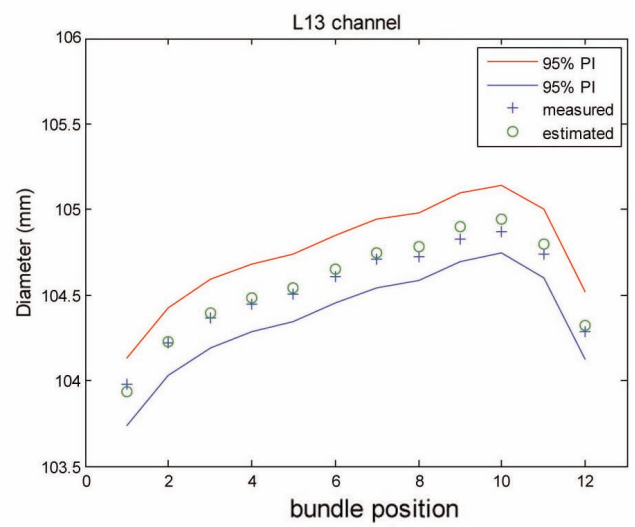

(d) L13 Channel (U2, 1944 EFPD)

Fig. 5. Prediction Intervals for Test Data 


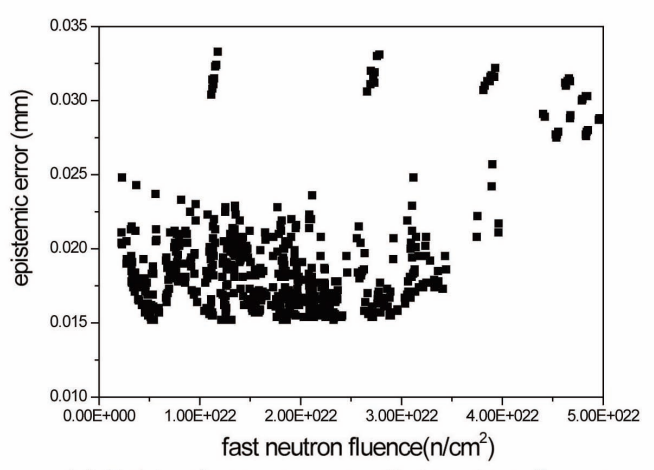

(a) Epistemic errors versus fast neutron fluence

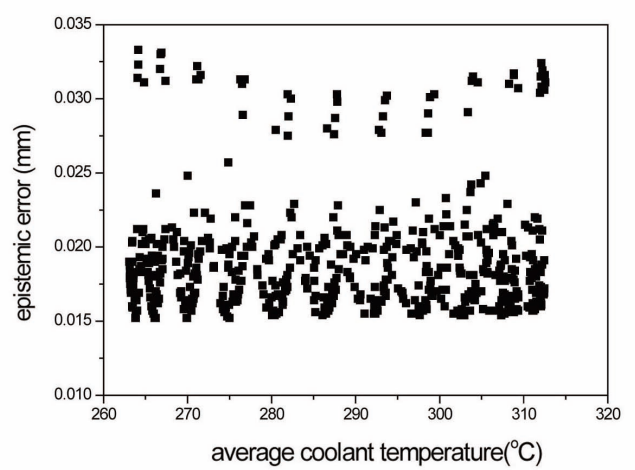

(b) Epistemic errors versus average coolant temperature

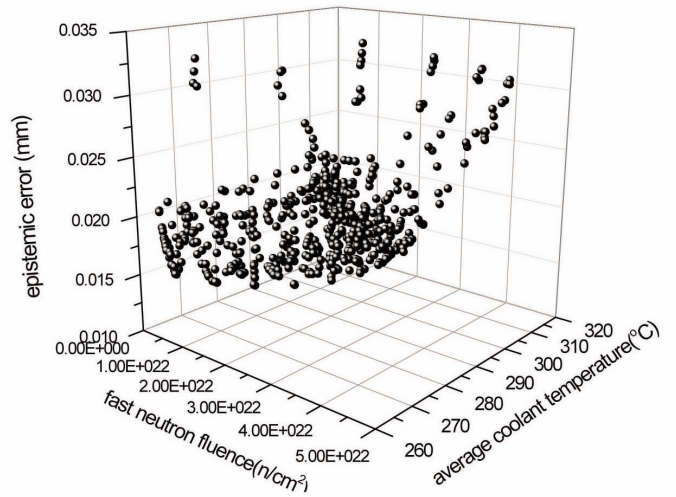

(c) $3 \mathrm{D}$ plot

Fig. 6. Epistemic Errors as a Function of Fast Neutron Fluence and Average Coolant Temperature

\section{CONCLUSIONS}

In this paper, a BPLM was developed to predict the PT diametral creep rate in CANDU reactors. Bundle position-wise biases were proposed in order to reduce prediction errors. The BPLM was developed using a data set (39 channels' data) prepared for training, and was tested using test data sets (10 channels' data) that differed from the training data. The SC method was also proposed to select the training data. The RMS errors of the PT diametral creep by the BPLM were less than those by the current design methodology. Uncertainty analysis results show that acceptable results are obtained, as the coverage exceeds 95\%. Therefore, the BPLM can be considered to be reliable based on these results.

In summary, a BPLM, which is based on a data based model, with the MLE technique to optimize the model parameters, is proposed for predicting the diameter of PT. The proposed BPLM with the MLE method has better capability for predicting the diameter of PT compared to the current mechanistic method.

\section{REFERENCES}

[ 1 ] N. Christodoulou et al., "Modeling In-Reactor Deformation of $\mathrm{Zr}-2.5 \mathrm{Nb}$ Pressure Tubes in CANDU Power Reactors," Zirconium in the Nuclear Industry: Eleventh International Symposium, ASTM STP 1295, E. R. Bradley and G. P. Sabol, Eds., American Society for Testing and Materials, pp. 518-537, 1996.

[2] D. Quach, P. Sermer, B. Phan, and O. Nainer, "Improvements in pressure tube diametral creep predictions and statistical error modeling," 28th Annual CNS Conference \& 31 SNS /CAN Student Conference, June 3-6, 2007, Saint John, New Brunswick, Canada, 2007.

[ 3 ] F.A. Garner, C.H. Henager and N. Igata, "Influence of Radiation on Material Properties," 13th International Symposium, Part 2, ASTM Special Technical Publication, 1987.

[4] D. Quach, Development of a Wolsong Units 2, 3, and 4 Best-Estimate with Uncertainty Pressure Tube Diametral Creep Model, KE001/RP/001 R00, June 30, 2010.

[5] S. L. Chiu, "Fuzzy model identification based on cluster estimation,” J. Intell. Fuzzy Systems, vol. 2, pp. 267-278, 1994. 